\title{
Short-Term Results of Total Knee Arthroplasty with Anterior-Posterior Glide LCS Mobile-Bearing System
}

\author{
Kyung Taek Kim, $\mathrm{MD}^{1}$, Min Soo Kang, $\mathrm{MD}^{1}$, Young Hoon Lim, $\mathrm{MD}^{2}$, Jin Woo Park, $\mathrm{MD}^{3}$, and Lih Wang, $\mathrm{MD}^{3}$ \\ ${ }^{1}$ Department of Orthopaedic Surgery, Dong-Eui Medical Center, Busan; ${ }^{2}$ Department of Orthopaedic Surgery, Busan Bon Hospital, Busan; ${ }^{3}$ Department of Orthopaedic \\ Surgery, Dong-A Medical Center, Busan, Korea
}

Purpose: To evaluate the clinical and radiological results of total knee arthroplasty (TKA) using the anterior-posterior glide (APG) low contact stress (LCS) mobile-bearing system.

Materials and Methods: We evaluated 130 knees in 117 patients who had undergone TKA with APG LCS mobile-bearing system between September 2005 and July 2007 and could be followed over 5 years. The mean follow-up period was 68 months. The clinical and radiological results were evaluated using the American Knee Society Scoring System, Oxford knee score and the American Knee Society Roentgenographic Evaluation and Scoring System. And we analyzed short-term postoperative complications.

Results: The average range of motion of the knee joint was $107.9^{\circ}$ (range, $70^{\circ}$ to $135^{\circ}$ ) preoperatively and $125.2^{\circ}\left(\right.$ range, $90^{\circ}$ to $135^{\circ}$ ) at the last followup. The average knee and functional scores were improved from 39.1 and 42.0 to 71.2 and 75.6, respectively, between the preoperative and last followup evaluation. The Oxford knee score was decreased from 42.9 preoperatively to 23.1 at the last follow-up. The femoro-tibial angle (anatomical axis) changed from $10.1^{\circ}$ varus preoperatively to $3.3^{\circ}$ valgus at the last follow-up. Radiolucency was observed in $14 \%$ of all cases. There were 1 case of traumatic dislocation of the polyethylene liner, 1 case of aseptic loosening and 6 cases of posterior instability because of posterior cruciate ligament (PCL) insufficiency.

Conclusions: TKA with APG LCS mobile-bearing system demonstrated relatively good short-term clinical and radiological results. However, further considerations for posterior instability associated with PCL insufficiency are needed.

Keywords: Knee, Arthroplasty, Anterior-posterior glide, Low contact stress, Mobile-bearing, Posterior instability

\section{Introduction}

Total knee arthroplsty (TKA) was first introduced in the 1960s as a new modality for pain relief and functional improvement in patients with degenerative arthritis or rheumatoid arthritis. So far, there has been much improvement in the TKA and various types of implant prostheses have been developed for the surgical procedure ${ }^{1-3)}$. Of those, the New Jersey low contact stress

Received October 30, 2013; Revised (1st) March 5, 2014;

(2nd) April 1, 2014; (3rd) June 5, 2014; Accepted June 8, 2014

Correspondence to: Lih Wang, MD

Department of Orthopaedic Surgery, Dong-A Medical Center, 26

Daesingongwon-ro, Seo-gu, Busan 602-715, Korea

Tel: +82-51-240-2593, Fax: +82-51-254-6757

E-mail: libi33@dau.ac.kr

This is an Open Access article distributed under the terms of the Creative Commons Attribution Non-Commercial License (http://creativecommons.org/licenses/by-nc/3.0/) which permits unrestricted non-commercial use, distribution, and reproduction in any medium, provided the original work is properly cited.
(LCS) mobile-bearing system designed to improve conformity and allow low constraint forces has been widely used since introduced by Buechel and Pappas ${ }^{4,5)}$ as an alternative to the fixedbearing system associated with component wear and loosening. In particular, the anterior-posterior glide LCS (APG LCS; DePuy Orthopaedics Inc., Warsaw, IN, USA) system is a modification of the previous rotating-platform (RP) system, which is equipped with a control arm for unrestricted anteroposterior range of motion. Theoretically, it facilitates effective femoral rollback when the posterior cruciate ligament (PCL) remains functional, which further allows multidirectional movement and thereby emulates close to normal knee kinematics ${ }^{6}$. In this study, we evaluated the short-term clinical and radiological results of TKA using the APG LCS system and investigated postoperative complications.

\section{Materials and Methods}

This study was approved by the Institutional Review Board of the Clinical Research Center of Dong-a University Hospital. 
Table 1. Patient Demographics

\begin{tabular}{lc}
\hline \multicolumn{1}{c}{ Parameter } & Mean (range) \\
\hline No. of knees & 130 \\
No. of patients & 117 \\
Duration of follow-up (mo) & $68(62-84)$ \\
Age $(\mathrm{yr})$ & $70.3(52-78)$ \\
Gender $(\mathrm{M}: \mathrm{F})$ & $13: 104$ \\
Height $(\mathrm{cm})$ & $156(140-188)$ \\
Weight $(\mathrm{kg})$ & $64(41-90)$ \\
Body mass index $\left(\mathrm{kg} / \mathrm{m}^{2}\right)$ & $26.4(16.7-37.5)$ \\
Bilateral knee status & \\
Other replaced & 23 \\
Two staged & 28 \\
Simultaneous & 18 \\
\hline
\end{tabular}

Between September 2005 and July 2007, 162 cases of TKA were performed using the APG LCS Complete (DePuy Orthopaedics Inc.) system at our institution. Of those, 117 patients (130 cases) who were available for $\geq 5$ years of follow-up were enrolled in this prospective study. The mean follow-up period was 68 months (range, 62 to 84 months). The excluded 25 patients (32 cases) were either lost to follow-up or unavailable for regular followup due to death ( 3 patients, 4 cases), unmet inclusion criteria (6 patients with rheumatoid arthritis, 9 cases), change of residence (12 patients, 15 cases), and advanced age and severe internal diseases ( 4 patients, 4 cases). All of the patients were female except for 13 males, and the mean age of the total study population was 70.3 years of age (range, 52 to 78 years) (Table 1). The indication for TKA was degenerative arthritis in all cases, and rheumatoid arthritis was an exclusion criterion. The inclusion criteria were the presence of an intact posterior PCL, $\leq 20^{\circ}$ varus deformity, $\leq 15^{\circ}$ valgus deformity, and $\leq 30^{\circ}$ flexion contracture. Patients with a knee joint infection or a history of TKA or trauma-related surgery were excluded from this study.

All the operations were performed by the same surgeon. A longitudinal midline skin incision was made over the knee joint and the joint capsule was incised through a medial parapatellar approach. Soft tissues including the ligaments and joint capsule were released, and the infrapatellar fat pad was removed in all cases. During bone resection, a gap technique was used for flexion-extension gap balancing. Proximal tibial resection was performed using an extraedullary alignment guide with the posterior tibial slope set at $3^{\circ}-5^{\circ}$. Distal femoral resection was done with an intramedullary alignment guide positioned to create a $5^{\circ}$ valgus angle, or a greater angle in the severe varus knee.
Patellar resurfacing was not carried out in all cases. Although the integrity of the PCL was assessed in the preoperative physical examination, the APG LCS system was used only when the ligament was intraoperatively confirmed to be intact with naked eyes and maintain normal function. In all cases, the femoral and tibial components were fixed with cement. Before closure of the surgical site, a physical examination was done to confirm the presence of $\leq 5 \mathrm{~mm}$ anteroposterior instability. Quadriceps femoris exercises were started from postoperative day one. Drainage was removed 2-3 days after surgery depending on the wound condition and knee joint exercises using continuous passive motion machine were initiated. Weight-bearing was allowed when active lower limb elevation was possible. Ambulation with a walking aid was commenced on the 4 th-7th postoperative day.

In principle, the assessments were performed before surgery and at 3 months, 1 year, 3 years, and 5 years after surgery. Although not all patients did attend every follow-up visit, the final 5-year postoperative assessment was conducted in all patients. Clinical evaluation was based on the American Knee Society Scoring System (knee score and functional score $)^{7)}$ and Oxford knee score ${ }^{8)}$ for subjective satisfaction rating. The knee score was measured on a 100-point scale to assess knee pain, joint stability, range of motion, flexion deformity, and joint deviation angle. The functional score was rated on a 100-point scale to assess the ability to walk and climb up stairs. Joint stability was assessed by physical examination, which was followed by roentgenographic stress test, if excessive anteroposterior instability or posterior tibial sagging was noted, to determine the level of instability. A $\geq 5 \mathrm{~mm}$ difference in the posterior translation distance on the lateral and posterior stress radiographs taken with the knee in $90^{\circ}$ flexion was considered as mild, $\mathrm{a} \geq 10 \mathrm{~mm}$ difference as moderate, and $\mathrm{a} \geq 15$ $\mathrm{mm}$ difference as severe. On the radiological evaluation, the location and fixation status of the implant and limb alignment were assessed according to the Knee Society Roentgenographic Evaluation and Scoring System ${ }^{9)}$ using preoperative and final follow-up anteroposterior and lateral standing views. To assess the implant location, the valgus angle of the femoral component $(\alpha)$ and the varus angle of the tibial component $(\beta)$ were measured on the anteroposterior view, and the flexion angle of the femoral component $(r)$ and the posterior tibial slope of the tibial component $(\sigma)$ were measured on the lateral view. The femorotibial angle (anatomical axis) was measured using the method of Bauer et al. ${ }^{10)}$ on the anteroposterior weight-bearing radiograph due to its relative convenience: the acute angle between the longitudinal axis drawn along the center of the femoral shaft and that drawn along the center of the tibial shaft was measured to determine varus/valgus 
alignment. On the implant fixation status assessment, the sum of radiolucent lines measured in $\mathrm{mm}$ in 7 regions on the lateral view was calculated for the femoral component, whereas the sum of radiolucent lines in 7 regions on the anteroposterior view and in 3 regions on the lateral view was calculated for the tibial component: a value of $\leq 4$ was considered suggesting non-progressive radiolucency; 5-9, requiring close observation; and $\geq 10$, indicating high probability of failure regardless of the presence of clinical symptoms ${ }^{11}$. Statistical analysis was done using SPSS ver. 12.0 (SPSS Inc., Chicago, IL, USA). The pre- and postoperative clinical score, range of motion, and femorotibial angle were compared using the paired $\mathrm{t}$-test.

\section{Results}

The mean range of motion was improved from $107.9^{\circ} \pm 17.4^{\circ}$ (range, $70^{\circ}$ to $135^{\circ}$ ) preoperatively to $125.2^{\circ} \pm 10.5^{\circ}$ (range, $90^{\circ}$ to $\left.135^{\circ}\right)$ at the last follow-up $(\mathrm{p}<0.001)$. The mean flexion contracture was $6.4^{\circ} \pm 6.8^{\circ}$ (range, $0^{\circ}$ to $30^{\circ}$ ) preoperatively and $2.5^{\circ} \pm 4.1^{\circ}$ (range, $0^{\circ}$ to $20^{\circ}$ ) at the last follow-up ( $\left.<<0.001\right)$. The mean knee score and functional score increased from $39.15 \pm 10.75$ points and $42.04 \pm 12.66$ points, respectively, to $71.24 \pm 13.92$ points and $75.58 \pm 17.72$ points, respectively, between the preoperative and last follow-up examinations. The Oxford knee score for subjective satisfaction decreased from a mean of $42.88 \pm 5.95$ points preoperatively to $23.09 \pm 5.78$ points at the last follow-up, indicating patients were satisfied with their results. The clinical improvements were statistically significant $(\mathrm{p}<0.001)$ (Fig. 1$)$.

The mean femorotibial angle was improved from $10.13^{\circ} \pm 4.92^{\circ}$ varus (range, $-25^{\circ}$ to $+2^{\circ}$ ) preoperatively to $3.31^{\circ} \pm 4.51^{\circ}$ valgus (range, $-4^{\circ}$ to $\left.+5^{\circ}\right)$ at the last follow-up $(\mathrm{p}<0.001)$. Regarding the implant location assessed according to the American Knee

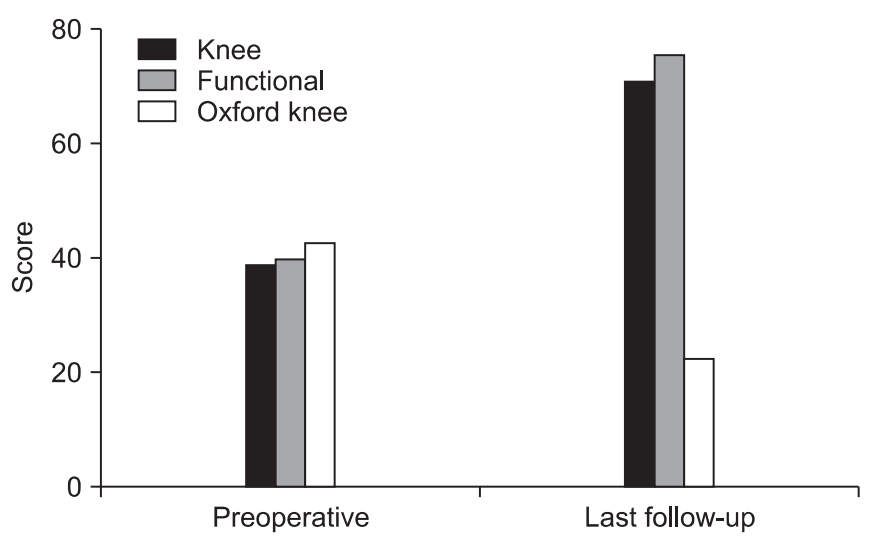

Fig. 1. The knee score, functional score and Oxford knee score were compared between the preoperative and last follow-up assessments.
Society Roentgenographic Evaluation and Scoring System, the mean $\alpha$ angle and $\beta$ angle on the anteroposterior view were $93.48^{\circ} \pm 4.96^{\circ}$ (range, $87^{\circ}$ to $100^{\circ}$ ) and $89.60^{\circ} \pm 3.17^{\circ}$ (range, $87^{\circ}$ to $94^{\circ}$ ), respectively. The mean $\gamma$ angle and $\delta$ angle on the lateral view were $3.6^{\circ} \pm 2.03^{\circ}$ (range, $0^{\circ}$ to $7^{\circ}$ ) and $86.86^{\circ} \pm 3.66^{\circ}$ (range, $80^{\circ}$ to $92^{\circ}$ ), respectively. Radiolucent lines were observed in 17 of 130 cases $(14 \%):<1 \mathrm{~mm}$ on the anteroposterior view of the tibia in 5 cases, $<1 \mathrm{~mm}$ on the lateral view of the femur in 9 cases, and $\geq 2 \mathrm{~mm}$ around the femoral component in 1 case and around the tibial component in 2 cases. The sum was less than 4 points in all of the 14 cases with $<1 \mathrm{~mm}$ radiolucent lines, showing no clinical significance. In the 3 cases with $\geq 2 \mathrm{~mm}$ radiolucent lines, except for the case with aseptic loosening, progression of radiolucency was not noted on the follow-up radiographs.

During the follow-up, revision due to polyethylene insert wear was not required in any cases, but polyethylene insert dislocation due to trauma occurred in 1 case, and aseptic loosening was noted in 1 case and posterior instability due to PCL insufficiency was observed in 6 cases. The polyethylene insert dislocation resulted from a slip and fall accident on the day of discharge from hospital. Dislocation of the $12.5-\mathrm{mm}$ polyethylene insert was accompanied by surgical site laceration, which was treated by debridement and irrigation and revision surgery using a $15-\mathrm{mm}$ polyethylene insert. The patient showed satisfactory results at
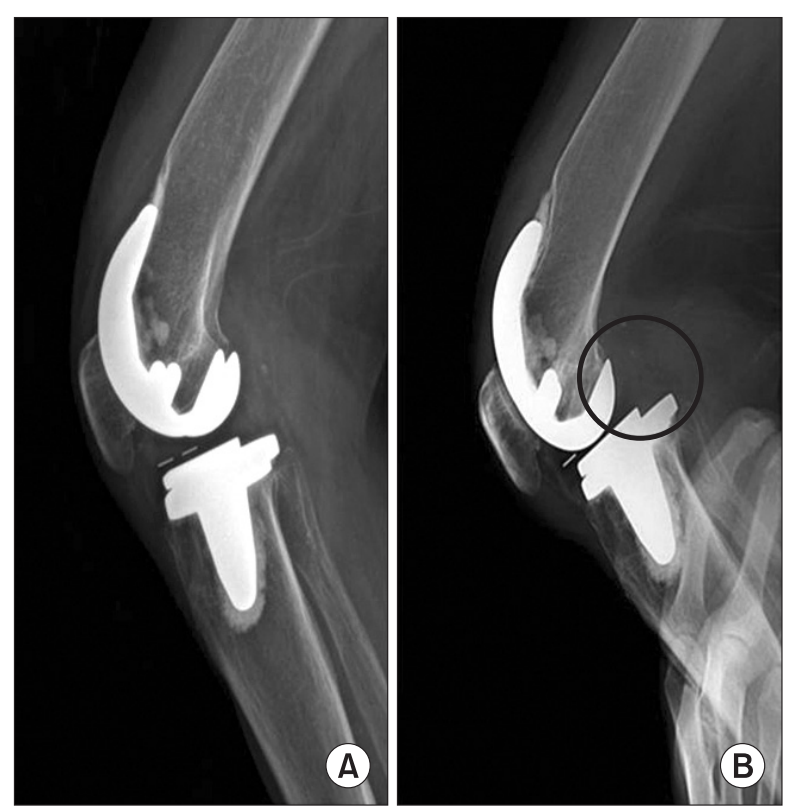

Fig. 2. (A) The plain lateral radiograph was obtained from a 64-year-old female who underwent total knee arthroplasty using anterior-posterior glide low contact stress prosthesis in the left knee due to osteoarthritis. (B) Posterior stress view demonstrated moderate (grade II; translation distance, $12 \mathrm{~mm}$ ) posterior instability at 40 months postoperatively. 
the final follow-up without any abnormal findings. The aseptic loosening that was identified at the 3rd postoperative year followup examination appeared to have progressed at the 5th postoperative year follow-up, but it has been kept under observation without treatment due to the absence of symptoms. The presence of posterior instability was observed 1 year after surgery in 1 case, 3 years after surgery in 3 cases (Fig. 2), and 5 years after surgery in 2 cases, all of which did not show any sign of progression. The level of instability was mild in 3 cases, moderate in 2 cases, and severe in 1 case. We recommended surgical intervention for the latter 3 cases. However, they declined further surgical treatment because of the lack of difficulty in performing daily living activities and the advanced age. Thus, they have been kept under observation with proper conservative treatment involving muscle strengthening exercises and application of a brace.

\section{Discussion}

Comparison between the APG LCS system and the RP system, its previous model, has been demonstrated in many studies. Compared to the RP system that allows a limited range of anteroposterior and mediolateral gliding movement, a bar incorporated into the tibial component of the APG LCS system facilitates greater anteroposterior gliding movement and rotatory movement in the knee after TKA. Kim and $\mathrm{Kim}^{12)}$ reported there was no significant difference between the RP system and the APG LCS system in terms of clinical scores and range of motion in 190 patients at 5 years after TKA. In contrast, Aigner et al. ${ }^{13)}$ reported that the mean range of motion was greater after TKA using the RP system. The APG LCS system did not result in notable improvement in femoral rollback and range of motion. In our previous study, the mean postoperative range of motion was $123.7^{\circ}$ in knees with the RP system ${ }^{14)}$. In the current study, the value was $125.2^{\circ}$ in knees with the APG LCS at the last follow-up. Although it is difficult to make direct comparison with the previous study, the clinical results of the current study were relatively satisfactory and showed no remarkable difference with the results of TKA using the RP system described in our previous study. Although the mean final follow-up knee score (71.2 points) and functional score (75.6 points) were noticeably low compared to the other studies ${ }^{6,15)}$, the magnitude of improvement from preoperative scores was statistically significant. Considering that the range of motion at the final follow-up was comparable to that in other studies and the preoperative scores were also lower than those in other studies, we believe such low follow-up knee scores can be attributed to the subjective prejudice of the authors of the current study. In addition, we recorded sense of discomfort, which was experienced especially during stair climbing, as the presence of mild or occasional pain in the final follow-up interview, which was one of the major causes for point deduction. The pain occurring during climbing down stairs may have been associated with the characteristics of the implant. In our opinion, however, quadriceps atrophy resulting from the lack of exercises after surgery could have significantly contributed to the development of pain.

Component loosening after TKA that has been considered as one of the major causes of complication and treatment failure can be assessed by examining radiolucency on radiographs. The theoretical advantage of the mobile-bearing systems is to minimize the bone-implant stress while maximizing the joint congruity. Lee et al. ${ }^{15)}$ reported that the radiological results of TKA using APG LCS system were satisfactory at a mean of 63 months after surgery: the radiolucent line score was either $<4$ point or progression of the radiolucent lines was not observed in all patients. In a 7-year follow-up study by Hwang et al. ${ }^{6}$, although radiolucent lines were observed in 13 of the 183 cases (7.1\%) after TKA using the APG LCS system, they were $\leq 2 \mathrm{~mm}$ non-progressive lines except in 1 case with substantial osteolysis. Although the prevalence of radiolucent lines was relatively high (14\%) in this study, we believe it is difficult to attribute this to the use of APG LCS system. The radiolucent lines were not progressive in all but 1 case with aseptic loosening, and there was no case with significant loosening, such as implant subsidence or dislocation. In spite of this, we think a longer follow-up is necessary to confirm our analysis.

Despite the theoretical advantages of the unprecedented, distinctive design, the APG LCS system has been associated with various potential complications. Bauze et al. ${ }^{16)}$ noted anterior impingement in 28 of 400 cases after TKA using the APG LCS system, which they thought would be prevented with proper component modification or excision of soft tissues or fat pads. Hwang et al. ${ }^{6}$ ) suggested the need for design modification based on the observation of implant dislocation and impingement for a minimum 7-year follow-up. On the other hand, Morberg et al. ${ }^{17)}$ emphasized more on the importance of the PCL integrity and tensioning rather than the implant design in preventing excessive instability. In our study, there was no case of anterior impingement, which we believe could be attributable to complete excision of fat pads during surgery. Implant dislocation, one of the most common problems of the mobile-bearing polyethylene insert, was observed in 1 case; however, it was caused by trauma. In our previous study, polyethylene insert fracture or dislocation occurred in 3 cases (1\%) after TKA using the rotating platform ${ }^{14)}$, whereas only 1 case of traumatic dislocation of the polyethylene 
insert was observed in the current study. This may be because we made more efforts during surgery to balance the medial and lateral ligaments in flexion and extension and to preserve the PCL, which was effective in preventing excessive flexion gap formation that is primarily responsible for implant dislocation. In addition, the anteroposterior gliding of the APG LCS system on the sagittal plane and the resultant efficiency in femoral rollback could have contributed to offset the mechanisms of polyethylene insert dislocation related to excessive flexion gap and rotation by multidirectional kinematics, which we believe should be investigated in further studies.

Anteroposterior instability after TKA using the APG LCS system occurred in 6 of the 16 cases due to PCL insufficiency in a study by Morberg et al. ${ }^{17)}$ during a 1-year short-term follow-up and in 12 of the 183 cases in a 7-year follow-up study by Hwang et al. ${ }^{6}$. Although 5 of the 12 cases (2.7\%) in the latter study exhibited excessive instability, revision was not considered necessary. In the current study, instability related to PCL insufficiency was observed in 6 cases (4.6\%), 4 of which occurred within 3 years after surgery. In our opinion, although it is difficult to rule out the possibility that PCL degeneration was the cause, minor iatrogenic injury to the PCL during surgery could have been responsible for the joint instability. In particular, considering that it is unavoidable to perform an excessive proximal tibial cut in a severe varus knee and the instrument is designed to have a $7^{\circ}$ posterior slope, some extent of damage to the tibial attachment site should occur, which could eventually lead to functional insufficiency. Taking this into consideration, we excluded patients with $>20^{\circ}$ varus deformity from the study population. In addition, the RP was selectively used in knees with a narrow posterior flexion gap caused by tight PCL with a goal of obtaining $3^{\circ}-5^{\circ}$ posterior tibial slope to avoid resulting in an excessive flexion gap after PCL resection. In spite of this, posterior instability developed in the short-term follow-up in our study; thus, extra caution is advised in the selection of study population and surgical techniques. It is recommended to exclude patients with a history of PCL injury or preoperative posterior instability as much as possible and sacrifice the PCL in patients with severe joint deformity or progressive rheumatoid arthritis ${ }^{18)}$. In knees with intraoperative PCL damage or insufficiency, suturing or augmentation should not be carried out. It is also advised to use the RP system without PCL release in knees with a narrow flexion gap due to tight PCL. In the absence of established treatment principles for PCL instability, further long-term follow-up studies should be conducted to determine the best form of treatment. Conservative therapy has been considered the treatment of choice in most cases, whereas functional instability followed by repeated effusion, limited range of motion, or constant anterior knee pain should be treated with revision surgery where the implanted APG polyethylene insert is replaced by a RP polyethylene insert ${ }^{17)}$. In our study, conservative treatment involving muscle strengthening exercises was prescribed to patients with mild posterior instability, whereas conservative treatment using muscle strengthening exercises and a brace or surgical treatment was considered for patients with moderate or severe instability depending on the presence of clinical instability. The three cases with moderate or severe posterior instability did not present with considerable discomfort with daily living activities, and thus revision was not carried out.

The limitations of this study include that 1) not all of the patients were available for every follow-up session even though the study design was prospective, 2) the study analysis was based on the clinical and radiological data without addressing the kinematics of the mobile-bearing system, 3) the radiolucency between the bone and the implant was assessed from radiographs taken in different planes without using fluoroscopy, 4) there was no specific measurement method for posterior instability and the stress test was not conducted preoperatively or on the intact knee, thus comparison was not feasible, and 5) the follow-up period was relatively short considering that there are many mid-term and long-term follow-up studies on TKA using the APG LCS system. However, the significance of this study is that it provides analysis of the overall treatment results and complications of TKA using the APG LCS system, which would raise awareness of general considerations and precautions to take during the procedure and eventually contribute to the collection of data for further modifications of the implant.

\section{Conclusions}

The short-term results of TKA using the APG LCS system were relatively satisfactory; however, we believe more consideration should be taken regarding PCL insufficiency.

\section{Conflict of Interest}

No potential conflict of interest relevant to this article was reported.

\section{Acknowledgments}

This study was supported by research funds from Dong-A University. 


\section{References}

1. Colizza WA, Insall JN, Scuderi GR. The posterior stabilized total knee prosthesis. Assessment of polyethylene damage and osteolysis after a ten-year-minimum follow-up. J Bone Joint Surg Am. 1995;77:1713-20.

2. Dennis DA, Clayton ML, O'Donnell S, Mack RP, Stringer EA. Posterior cruciate condylar total knee arthroplasty. Average 11-year follow-up evaluation. Clin Orthop Relat Res. 1992;(281):168-76.

3. Buehler KO, Venn-Watson E, D'Lima DD, Colwell CW Jr. The press-fit condylar total knee system: 8- to 10-year results with a posterior cruciate-retaining design. J Arthroplasty. 2000;15:698-701.

4. Buechel FF, Pappas MJ. Long-term survivorship analysis of cruciate-sparing versus cruciate-sacrificing knee prostheses using meniscal bearings. Clin Orthop Relat Res. 1990;(260): 162-9.

5. Buechel FF, Pappas MJ. New Jersey low contact stress knee replacement system. Ten-year evaluation of meniscal bearings. Orthop Clin North Am. 1989;20:147-77.

6. Hwang BH, Lee WS, Park KK, Yang IH, Han CD. Anteriorposterior glide mobile-bearing total knee arthroplasty: complications related to prosthesis design. J Arthroplasty. 2011; 26:1438-44.

7. Insall JN, Dorr LD, Scott RD, Scott WN. Rationale of the Knee Society clinical rating system. Clin Orthop Relat Res. 1989;(248):13-4.

8. Dawson J, Fitzpatrick R, Murray D, Carr A. Questionnaire on the perceptions of patients about total knee replacement. J Bone Joint Surg Br. 1998;80:63-9.

9. Ewald FC. The Knee Society total knee arthroplasty roent- genographic evaluation and scoring system. Clin Orthop Relat Res. 1989;(248):9-12.

10. Bauer GC, Insall J, Koshino T. Tibial osteotomy in gonarthrosis (osteo-arthritis of the knee). J Bone Joint Surg Am. 1969;51:1545-63.

11. Plante-Bordeneuve P, Freeman MA. Tibial high-density polyethylene wear in conforming tibiofemoral prostheses. J Bone Joint Surg Br. 1993;75:630-6.

12. Kim YH, Kim JS. Comparison of anterior-posterior-glide and rotating-platform low contact stress mobile-bearing total knee arthroplasties. J Bone Joint Surg Am. 2004;86:123947.

13. Aigner C, Windhager R, Pechmann M, Rehak P, Engeleke $\mathrm{K}$. The influence of an anterior-posterior gliding mobile bearing on range of motion after total knee arthroplasty: a prospective, randomized, double-blinded study. J Bone Joint Surg Am. 2004;86:2257-62.

14. Kim KT, Kim SH, Jang HS. Total knee arthroplasty using LCS mobile bearing system. J Korean Knee Soc. 2004;16:1117.

15. Lee DH, Yang IW, Han CD. Total knee arthroplasty with anteroposterior glide low contact stress: minimum 5-year follow-up results. J Korean Orthop Assoc. 2006;41:650-7.

16. Bauze AJ, Falworth MS, Oakeshott RD. Early results of total knee arthroplasty with a low contact stress anteroposterior glide. J Orthop Surg (Hong Kong). 2009;17:174-8.

17. Morberg P, Chapman-Sheath P, Morris P, Cain S, Walsh WR. The function of the posterior cruciate ligament in an anteroposterior-gliding rotating platform total knee arthroplasty. J Arthroplasty. 2002;17:484-9.

18. Yim S, Seo Y, Jang M. Posterior cruciate ligament retaining total knee arthroplasty. J Korean Knee Soc. 2011;23:1-6. 